\title{
Wie sind Beschwerden nach Weingenuß allergologisch einzuordnen?
}

\author{
Imke Reese \\ Ernährungsberatung und -therapie Allergologie, München, Germany
}

\begin{abstract}
Unverträglichkeiten nach Wein-Genuß werden häufig berichtet [1,2]. Oftmals handelt es sich dabei um Untersuchungen, die auf der Auswertung von Fragebögen basieren. Nur selten lässt sich diesen Berichten entnehmen, ob es sich tatsächlich um ein reproduzierbares Phänomen handelt und damit definitionsgemäß eine Unverträglichkeit vorliegt. Eine fundierte Diagnostik zur Identifizierung des zugrundeliegenden Pathomechanismus sowie eine Objektivierung der Symptome fehlen zumeist, so dass entsprechende Berichte oftmals nur die subjektive Wahrnehmung der Betroffenen widerspiegeln. Auch im klinischen Alltag liegt meist nur eine Verdachtsdiagnose aufgrund der Krankengeschichte oder der Eigenverdacht des Betroffenen vor.
\end{abstract}

Als mögliche Auslöser werden vor allem Sulfit und biogene Amine angeschuldigt. Sulfit gilt als maßgeblicher Auslöser für respiratorische Reaktionen, biogene Amine können für Symptome an mehreren Organsystemen verantwortlich sein. Doch die wenigen vorliegenden Provokationsstudien konnten weder Sulfit noch biogene Amine als eindeutige Auslöser für Weinbzw. alkoholabhängige Reaktionen identifizieren [3-8]. Als potentielle Auslöser bzw. als Ursache für Beschwerden nach Weingenuß sind durchaus noch weitere Substanzen bzw. Pathomechanismen möglich: Selbst schwere anaphylaktische Reaktionen gegenüber Traubenallergenen bzw. dem Alkohol selbst sind beschrieben [9,10]. Mit der EU-weiten Deklarationspflicht für häufige und potente Allergene sind zudem gängige Schönungsmittel auf Basis von Milch, Ei und Fisch als mögliche Auslöser ins Visier geraten [11]. Die klinische Relevanz ist allerdings nach wie vor sehr fraglich [12], zumal Milch- und Eiallergien typischerweise im Kleinkindalter auftreten und bereits bei Eintritt in die Schule bei den meisten Betroffenen nicht mehr bestehen.
Weingenuss kann über ganz verschiedene Pathomechanismen Beschwerden auslösen. Allerdings handelt es sich nicht immer um eine Unverträglichkeit im allergologischen Sinne. Nur eine fundierte Diagnostik - inkl. der Auswertung von Ernährungsprotokollen - ermöglicht den Nachweis einer tatsächlichen Unverträglichkeit und die Identifizierung des zugrundeliegenden Pathomechanismus. Die Kenntnis des verantwortlichen Auslösers und der Art der Unverträglichkeit bilden die Grundlage für eine sinnvolle Therapie, ohne die Lebensqualität des Betroffenen unnötig einzuschränken.

[1] P. Wigand, Dtsch Arztebl Int, 109, 437 (2012)

[2] J.C. Cardet, J Allergy Clin Immunol Pract, 2, 208 (2014)

[3] H. Vally, J Allergy Clin Immunol, 103, 41 (1999)

[4] H. Vally, and P.J. Thompson, Addict Biol, 8, 3 (2003)

[5] H. Vally, P.J. Thompson and N.L. Misso, Clin Exp Allergy, 37, 1062 (2007)

[6] I. Ehlers, Clin Exp Allergy, 32, 1231 (2002)

[7] G. Kanny, J Allergy Clin Immunol, 107, 375 (2001)

[8] F. Wantke, Int Arch Allergy Immunol, 110, 397 (1996)

[9] S.G. Schad, World Allergy Organ J, 3, 1 (2010)

[10] S.L. Fernando and L.R. Clarke, Case Rep Dermatol, 1, 1 (2009)

[11] M. Deckwart, Food Addit Contam Part A Chem Anal Control Expo Risk Assess, 31, 1805 (2014)

[12] E. Vassilopoulou, Clin Transl Allergy, 1, 10 (2011)

This is an Open Access article distributed under the terms of the Creative Commons Attribution License 4.0, which permits unrestricted use, distribution, and reproduction in any medium, provided the original work is properly cited. 